\title{
Reentrance of the Disordered Phase in the Antiferromagnetic Ising Model on a Square Lattice with Longitudinal and Transverse Magnetic Fields
}

\author{
Ryui Kaneko, ${ }^{*}$ Yoshihide Douda, Shimpei Goto,$^{\dagger}$ and Ippei Danshita ${ }^{\ddagger}$ \\ Department of Physics, Kindai University, Higashi-Osaka, Osaka 577-8502, Japan
}

(Dated: June 10, 2021)

\begin{abstract}
Motivated by recent experiments with Rydberg atoms in an optical tweezer array, we accurately map out the ground-state phase diagram of the antiferromagnetic Ising model on a square lattice with longitudinal and transverse magnetic fields using the quantum Monte Carlo method. For a small but nonzero transverse field, the transition longitudinal field is found to remain nearly constant. By scrutinizing the phase diagram, we uncover a narrow region where the system exhibits reentrant transitions between the disordered and antiferromagnetic phases with increasing transverse field. Our phase diagram provides a useful benchmark for quantum simulation of a Rydberg atom system.
\end{abstract}

Quantum effects in many-body systems have been the subject of intensive research. Accurate simulation of quantum systems can reveal novel phases and phase transitions. Although numerical simulation on a classical computer is useful, the number of tractable models is limited because of the exponential growth of the Hilbert space. An alternative approach is to use highly controllable devices, namely, analog quantum simulators, to emulate quantum many-body systems [1-3].

Quantum simulators using Rydberg atoms in an optical tweezer array have attracted growing interest owing to rapid technological advances [4]. Optical tweezers allow one to hold and move each atom precisely. In addition, the distance between atoms is large enough that individual atoms can be observed. Because dipole-dipole interactions between Rydberg atoms are much stronger than those between ground-state atoms, one can conduct experiments at relatively high temperatures without evaporative cooling, and the typical time scale of the real-time dynamics is roughly 1000 times faster than that of ultracold atoms in optical lattices.

Owing to these advantages, recent experiments using Rydberg atom arrays [5-12] have successfully observed various interesting many-body phenomena. For example, quantum phase transitions and nonequilibrium dynamics [5, 6] have been observed in Rydberg systems that realize the onedimensional Ising model with longitudinal and transverse magnetic fields. Furthermore, symmetry-protected topological phases have been identified in a simulator that imitates the $\mathrm{Su}-$ Schrieffer-Heeger chain [12]. The simulation of not only systems in one spatial dimension but also those in two spatial dimensions is feasible [7,8]. Very recently, the number of controllable atoms exceeded $200[9,10]$.

The recent development of quantum simulation experiments has motivated a revival of theoretical research on fundamental quantum spin models. In particular, the study of nonequilibrium dynamics is among the most active fields. For instance, the observation of certain states that exhibit anomalously slow thermalization $[5,11]$ has stimulated research on quantum

\footnotetext{
* rkaneko@ phys.kindai.ac.jp

† goto.las@tmd.ac.jp; Present address: College of Liberal Arts and Sciences, Tokyo Medical and Dental University, Ichikawa, Chiba 272-0827, Japan

$\ddagger$ danshita@phys.kindai.ac.jp
}

many-body scars [13-16]. There are many open questions on how quantum information propagates in terms of the real-time dynamics of the quantum Ising model [7, 8].

It is essential to understand the ground-state properties of static systems before tackling these unresolved problems. The Ising model has served as a textbook example of how to describe a phase transition in statistical physics $[17,18]$ because of its simplicity and solvability [19]. Rydberg systems are suitable for realizing the quantum Ising model. In these systems, the longitudinal and transverse fields can be controlled by frequency detuning and the Rabi frequency of the laser, respectively [20]. The ground-state phase diagrams of quantum Ising models on several lattices have been extensively studied using the quantum Monte Carlo (QMC) method [21-23].

Although many Ising models have been analyzed, the precise ground-state properties of the mixed-field Ising model have yet to be explored on the simple square lattice. The model is so primitive that detailed analysis has been overlooked. In one spatial dimension, the precise phase diagram of the mixedfield Ising model is determined by the exact diagonalization (ED) [24, 25], QMC [26], and density matrix renormalization group [27] methods. By contrast, in two spatial dimensions, only a schematic phase diagram for a few dozen sites has been drawn in a recent ED study [8].

In this letter, we draw the ground-state phase diagram of the antiferromagnetic Ising model on a square lattice with both longitudinal and transverse fields. The Hamiltonian of the mixed-field Ising model is defined as

$$
H=J \sum_{\langle i, j\rangle} \hat{S}_{i}^{z} \hat{S}_{j}^{z}-h \sum_{i} \hat{S}_{i}^{z}-\Gamma \sum_{i} \hat{S}_{i}^{x},
$$

where $J(>0)$ denotes the strength of the antiferromagnetic Ising interaction, and $h(\Gamma)$ represents the longitudinal (transverse) magnetic field. The operators $\hat{S}^{z}$ and $\hat{S}^{x}$ are the $z$ and $x$-component $S=1 / 2$ Pauli spin operators. The notation $\langle i, j\rangle$ indicates that sites $i$ and $j$ are nearest neighbors. We set $\hbar=k_{\mathrm{B}}=a=1$ and take $J$ as the unit of energy, where $a$ is the lattice spacing.

We used the QMC method to draw the ground-state phase diagram of the mixed-field Ising model on a square lattice. We adopted the Discrete Space Quantum Systems Solver (DSQSS) library [28], which implements the directed loop algorithm [29]. We chose the periodic-periodic boundary con- 


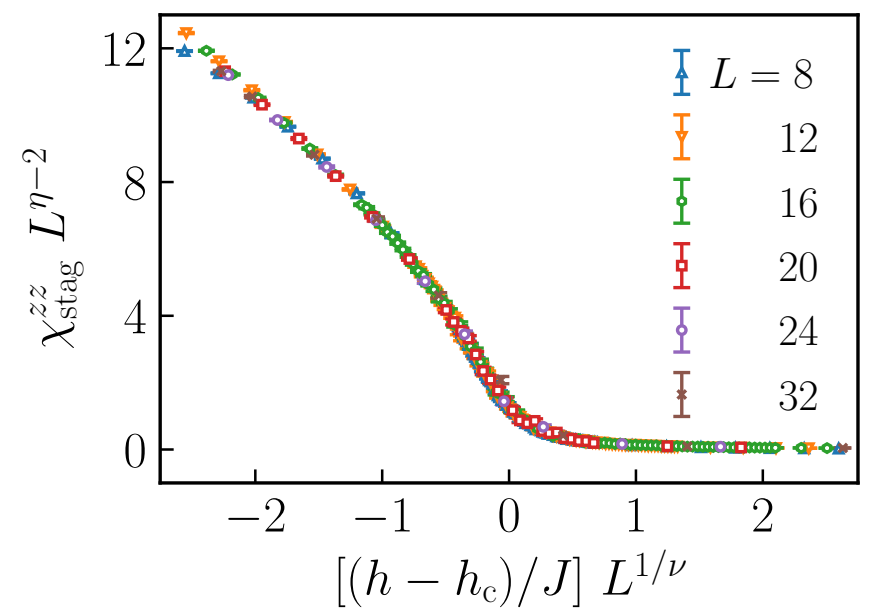

FIG. 1. (Color online) Finite-size scaling analysis at $\Gamma / J=0.1$ of the staggered magnetic susceptibility. Because the transition belongs to the Ising universality class, we choose $\eta=0.03631$ (3) and $v=0.62999(5)$ [30]. We also fixed the inverse temperature $\beta J / L=8$ assuming $z=1$. The transition field is estimated as $h_{\mathrm{c}} / J=2.00426(3)$.

dition and considered the system sizes $N_{\mathrm{s}}=L^{2}$ with $L \leq 32$. We typically performed $10^{5}$ Monte Carlo steps to observe the physical quantities after discarding $10^{5}$ Monte Carlo steps for thermalization. The statistical average was taken over 64 independent runs.

To characterize each phase, we calculated the staggered magnetic susceptibility [31], which is defined as

$$
\begin{gathered}
\chi_{\text {stag }}^{z z}=\frac{\left\langle\hat{M}^{z}(\boldsymbol{Q})^{2}\right\rangle}{\beta L^{d}}, \quad Q=(\pi, \pi), \\
\text { and } \hat{M}^{z}(\boldsymbol{q})=\int_{0}^{\beta} d \tau \sum_{j} \hat{S}_{j}^{z}(\tau) e^{-i \boldsymbol{q} \cdot \boldsymbol{r}_{j}},
\end{gathered}
$$

where $\boldsymbol{r}_{j}$ is the real space coordinate at site $j, d(=2)$ is the spatial dimension, and $\beta$ is the inverse temperature. When $h$ and $\Gamma$ are smaller than the critical values, the ground state is antiferromagnetic, and the staggered magnetic susceptibility $\chi_{\text {stag }}^{z z}$ diverges as $\beta L^{d}$ for sufficiently large $L$ and $\beta$. By contrast, when $h$ or $\Gamma$ is larger than the critical value, the ground state is disordered, and $\chi_{\text {stag }}^{z z}$ is upper bounded.

We performed finite-size scaling analysis based on Bayesian scaling analysis [32] to determine the phase boundary between the antiferromagnetic and disordered phases at zero temperature. We set the inverse temperature $\beta$ to be proportional to the linear system size $L$ because the dynamical exponent would satisfy $z=1$. The scaling form [31] of $\chi_{\text {stag }}^{z z}$ is given as

$$
\chi_{\text {stag }}^{z z} \sim L^{2-\eta} \mathcal{F}\left(\delta L^{1 / v}\right),
$$

where $\mathcal{F}$ is a scaling function, $\eta$ is the anomalous dimension, and $v$ is the correlation length exponent. The difference of the field from the critical point is written as $\delta=\left(h-h_{\mathrm{c}}\right) / J[\delta=$ $\left.\left(\Gamma-\Gamma_{\mathrm{c}}\right) / J\right]$ for a fixed $\Gamma(h)$. Because the continuous transition for $\Gamma>0$ is expected to belong to the Ising universality class,

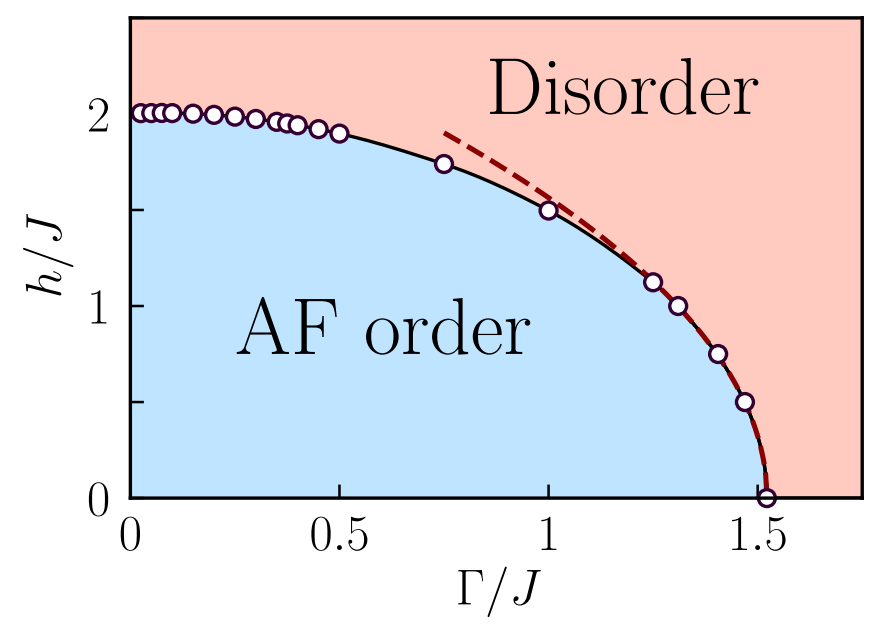

FIG. 2. (Color online) Ground-state phase diagram of the antiferromagnetic Ising model on a square lattice with longitudinal and transverse magnetic fields. The statistical error is smaller than the symbol size. We obtained the transition point at $h=0$ as $\Gamma_{\mathrm{c}}(h=0)=1.5220(4)$, which is consistent with previous QMC studies $[21,33]$. The transition longitudinal field $h_{\mathrm{c}}$ changes very little and remains $h_{\mathrm{c}} \sim 2 J$ for small $\Gamma$. The dashed line corresponds to asymptotic behavior of the phase boundary obtained by the MF approximation [27], namely, $\Gamma_{\mathrm{c}} \sim \Gamma_{\mathrm{c}}(h=0)+c_{2} h^{2} /(2 J)$. Here the coefficient obtained by fitting is given as $c_{2}=-0.427(3)$, whereas $\Gamma_{\mathrm{c}}(h=0)$ is chosen as the transition point in two spatial dimensions.

we set the critical exponents to those in the $(2+1) \mathrm{D}$ Ising model, namely, $\eta=0.03631$ (3) and $v=0.62999$ (5) [30]. The transition field $h_{\mathrm{c}}\left(\Gamma_{\mathrm{c}}\right)$ was estimated for each $\Gamma(h)$. Figure 1 shows an example of the finite-size scaling analysis at $\Gamma / J=$ 0.1 . The data for different system sizes collapse onto a single curve at $h \sim h_{\mathrm{c}}$.

We obtain the ground-state phase diagram shown in Fig. 2. At $\Gamma=0$, the model becomes classical and is known to exhibit a first-order transition at $h_{\mathrm{c}}=d J$ [24-27, 31]. Our numerical data are consistent with this finding; for small $\Gamma$, the transition longitudinal field changes very little and remains $h_{\mathrm{c}} \sim 2 J$. By contrast, for $h \sim 0$, the transition transverse field $\Gamma_{\mathrm{c}}$ decreases very sharply. This observation is similar to the results of the mean-field (MF) approximation [27], which corresponds to the limit of infinite spatial dimensions, as well as that in one spatial dimension [24-27]. The phase boundary satisfies $\left[\Gamma_{\mathrm{c}}-\right.$ $\left.\Gamma_{\mathrm{c}}(h=0)\right] /(d J) \sim c_{d}[h /(d J)]^{2}$ with $c_{1} \sim-0.37$ [24-27] $\left(c_{\infty}=-0.375\right.$ [27]) in one spatial dimension (infinite spatial dimensions). We found that $c_{2}=-0.427(3)$ in two spatial dimensions. The coefficients $c_{1}, c_{2}$, and $c_{\infty}$ are approximately -0.4 . There seems to be no significant difference between these values irrespective of the spatial dimensions.

Figure 3 shows the magnified ground-state phase diagram for $h \sim 2 J$ and $\Gamma \sim 0$. Remarkably, we found a narrow region where the disordered phase exhibits reentrance when the transverse field increases. Near $\Gamma=0$, the transition longitudinal field $h_{\mathrm{c}}$ increases as the transverse field $\Gamma$ increases. For $\Gamma \gtrsim 0.075, h_{\mathrm{c}}$ starts to decrease as $\Gamma$ increases. This observation is qualitatively different from that in one spatial dimension [24-27], where $h_{\mathrm{c}}$ decreases monotonically as $\Gamma$ 


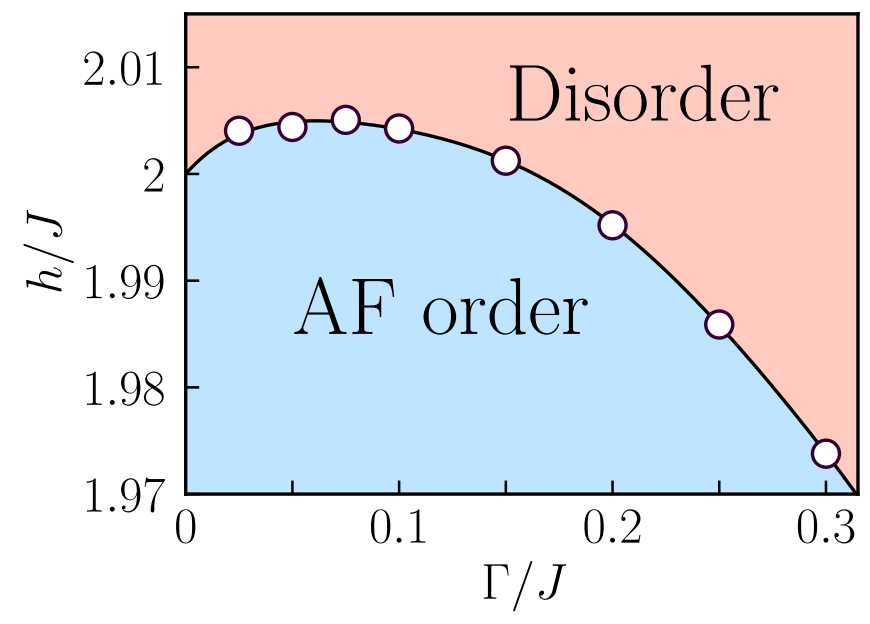

FIG. 3. (Color online) Magnified ground-state phase diagram near $\Gamma=0$, where the disordered phase exhibits reentrance. The statistical error is smaller than the symbol size. The curve is obtained by fitting the data to the sextic polynomial for $0 \leq \Gamma / J \leq 0.35$. For $0 \leq \Gamma / J \lesssim 0.075$, the transition longitudinal field $h_{\mathrm{c}}$ increases as the transverse field $\Gamma$ increases.

increases. The transverse field usually destabilizes the antiferromagnetic order in low spatial dimensions. Therefore, we expect a monotonic decrease in the transition field $h_{\mathrm{c}}$. However, this is not the case for the square Ising model.

Unconventional reentrant behavior has already been observed in the MF approximation [27], and our results show that it is maintained even in two spatial dimensions. Here we briefly review the MF results on the tilt of the spins and the variation of the longitudinal transition field in the presence of a small transverse field. The MF energy per site is given as $E /\left(d J N_{\mathrm{s}}\right)=$ $\left[\cos \theta_{1} \cos \theta_{2}-\tilde{\Gamma}\left(\sin \theta_{1}+\sin \theta_{2}\right)-\tilde{h}\left(\cos \theta_{1}+\cos \theta_{2}\right)\right] / 4$. Here $d J$ is taken as the unit of energy, and the fields are rescaled as $\tilde{\Gamma}=\Gamma /(d J)$ and $\tilde{h}=h /(d J)$. All spins lie in the $x z$ plane, and $\theta_{1}\left(\theta_{2}\right)$ is the angle between the $z$ axis and the spin at one (the other) sublattice site on a bipartite lattice. The critical point can be obtained using the stationary condition $-\sin \theta_{i} \cos \theta_{j}-\tilde{\Gamma} \cos \theta_{i}+\tilde{h} \sin \theta_{i}=0$ with $(i, j)=(1,2),(2,1)$. We would like to obtain the angle $\theta_{\mathrm{c}}(\tilde{\Gamma})$ at the critical point in the presence of a small transverse field $\tilde{\Gamma} \ll 1$. Let us define the relative angle between two spins as $\delta \theta=\theta_{2}-\theta_{1}$. At the critical point $\left(\tilde{h}=\tilde{h}_{\mathrm{c}}\right), \delta \theta=0$, and thus $\theta_{1}=\theta_{2}=\theta_{\mathrm{c}}$. Therefore, $\theta_{\mathrm{c}}$ satisfies

$$
-\sin \theta_{\mathrm{c}} \cos \theta_{\mathrm{c}}-\tilde{\Gamma} \cos \theta_{\mathrm{c}}+\tilde{h} \sin \theta_{\mathrm{c}}=0 .
$$

Slightly away from the critical point, we can expand the stationary condition up to the first order of $\delta \theta \ll 1$. Replacing $\theta_{1}$ with $\theta_{\mathrm{c}}$, we obtain

$$
1-\tilde{\Gamma} \sin \theta_{\mathrm{c}}-\tilde{h} \cos \theta_{\mathrm{c}}=0 .
$$

Eliminating $\tilde{h}$ from Eqs. (5) and (6) yields

$$
\sin ^{3} \theta_{\mathrm{c}}=\tilde{\Gamma} .
$$

Without the transverse field $\tilde{\Gamma}$, the spins align in the $z$ direction, and the angle at $\tilde{h}=\tilde{h}_{\mathrm{c}}+0$ is $\theta_{\mathrm{c}}=0$. For small $\tilde{\Gamma}$, Eq. (7) suggests that the tilting angle grows more rapidly than it does in a linear field $\left(\theta_{\mathrm{c}} \sim \tilde{\Gamma}^{1 / 3}\right)$. The $z$ component ( $x$ component) of the spin becomes significantly smaller (larger). To compensate for the smaller $z$ component, the longitudinal field should be sufficiently large to cause the transition; that is, $\tilde{h}_{\mathrm{c}}(\tilde{\Gamma} \ll 1)>$ $\tilde{h}_{\mathrm{c}}(\tilde{\Gamma}=0)$. Indeed, from Eqs. (6) and (7), the transition field satisfies $\tilde{h}_{\mathrm{c}}=\left(1-\tilde{\Gamma}^{2 / 3}\right)^{1 / 2}\left(1+\tilde{\Gamma}^{2 / 3}\right) \sim 1+\tilde{\Gamma}^{2 / 3} / 2$, which clearly indicates reentrance.

As we see below, subtle competition between the MF mechanism and quantum fluctuations determines whether reentrance occurs. The ground state at $(\Gamma, h)=(0, d J)$ is macroscopically degenerate $[24,27,31,34-36]$. The first-order transition at this point becomes a continuous one in the presence of an infinitesimally small $\Gamma$. The shape of the phase boundary near $\Gamma=0$ is susceptible to quantum fluctuations, which can vary depending on the spatial dimensions of the system. In one spatial dimension, strong quantum fluctuations destabilize the antiferromagnetic order. The transition longitudinal field behaves as $h_{\mathrm{c}} \sim J+c_{1}^{\prime} \Gamma$ with a negative coefficient $c_{1}^{\prime} \sim-0.68(4)$ [27, 37-39]. By contrast, in the MF approximation, which can be regarded as the limit of infinite spatial dimensions, the antiferromagnetic order is favored. The transition obeys the relation $h_{\mathrm{c}} \sim J+c_{\infty}^{\prime} \Gamma^{2 / 3}$ with a positive coefficient $c_{\infty}^{\prime}=0.5$ [27]. On a square lattice, we found numerically that the transition satisfies $h_{\mathrm{c}} \sim 2 J+c_{2}^{\prime} \Gamma$ with a very small positive coefficient, $c_{2}^{\prime} \sim 0.16$. Here we assume that the longitudinal transition point is a linear function of the transverse field for $\Gamma \sim 0$. This behavior is predicted by perturbation theory in one spatial dimension [27], although it does not have to occur in general. The monotonic behavior of the coefficients $\left(c_{1}^{\prime}<c_{2}^{\prime}<c_{\infty}^{\prime}\right)$ suggests that the shape of the phase boundary in two spatial dimensions is intermediate between those in one and infinite spatial dimensions.

In conclusion, we studied the antiferromagnetic Ising model on a square lattice with longitudinal and transverse magnetic fields using the QMC method. We determined the phase boundary between the antiferromagnetic and disordered phases by finite-size scaling analysis and found that the critical field $h_{\mathrm{c}}$ changes very little for small $\Gamma$. We also found a narrow region where the disordered phase exhibits reentrance near the point $(\Gamma, h) \sim(0,2 J)$. By comparing our result with those of previous studies, we found that the shape of the phase boundary in two spatial dimensions is intermediate between those in one and infinite spatial dimensions. Reentrant behavior, which occurs in infinite spatial dimensions, seems to emerge in the rather low dimensionality of two spatial dimensions.

Our phase diagram would be helpful for analog quantum simulation of Rydberg systems. Although it is challenging to detect the narrow reentrant region on a square lattice, a nearly intact transition field $h_{\mathrm{c}}$ as a function of $\Gamma$ may be observed for $\Gamma \sim 0$. This behavior can be measured to confirm the accuracy of quantum simulations.

In three spatial dimensions, the MF critical exponent will be exact because the value $d+z=4$ reaches the upper critical dimension. We may observe a broader reentrant region. It is of great interest to investigate how the phase boundary is modified on a cubic lattice. This topic remains a subject of future study. In addition, atoms in Rydberg states are governed 
by the van der Waals interaction. It is also interesting to study the Ising model with more realistic long-range interaction [40] and related models in geometrically frustrated lattices [41-43]. This topic also remains a subject of future study.

\section{ACKNOWLEDGMENTS}

The authors acknowledge fruitful discussions with T. Uno. This work was financially supported by JSPS KAKENHI
(Grants Nos. 18K03492 and 18H05228), by JST CREST (Grant No. JPMJCR1673), and by MEXT Q-LEAP (Grant No. JPMXS0118069021). The numerical computations were performed on computers at the Yukawa Institute Computer Facility and on computers at the Supercomputer Center, the Institute for Solid State Physics, the University of Tokyo.
[1] R. P. Feynman, Int. J. Theor. Phys 21, 467 (1982).

[2] I. Buluta and F. Nori, Science 326, 108 (2009).

[3] I. M. Georgescu, S. Ashhab, and F. Nori, Rev. Mod. Phys. 86, 153 (2014).

[4] A. Browaeys and T. Lahaye, Nat. Phys. 16, 132 (2020).

[5] H. Bernien, S. Schwartz, A. Keesling, H. Levine, A. Omran, H. Pichler, S. Choi, A. S. Zibrov, M. Endres, M. Greiner, V. Vuletić, and M. D. Lukin, Nature 551, 579 (2017).

[6] A. Keesling, A. Omran, H. Levine, H. Bernien, H. Pichler, S. Choi, R. Samajdar, S. Schwartz, P. Silvi, S. Sachdev, P. Zoller, M. Endres, M. Greiner, V. Vuletić, and M. D. Lukin, Nature 568, 207 (2019)

[7] E. Guardado-Sanchez, P. T. Brown, D. Mitra, T. Devakul, D. A. Huse, P. Schauß, and W. S. Bakr, Phys. Rev. X 8, 021069 (2018).

[8] V. Lienhard, S. de Léséleuc, D. Barredo, T. Lahaye, A. Browaeys, M. Schuler, L.-P. Henry, and A. M. Läuchli, Phys. Rev. X 8, 021070 (2018).

[9] P. Scholl, M. Schuler, H. J. Williams, A. A. Eberharter, D. Barredo, K.-N. Schymik, V. Lienhard, L.-P. Henry, T. C. Lang, T. Lahaye, A. M. Läuchli, and A. Browaeys, arXiv:2012.12268 .

[10] S. Ebadi, T. T. Wang, H. Levine, A. Keesling, G. Semeghini, A. Omran, D. Bluvstein, R. Samajdar, H. Pichler, W. W. Ho, S. Choi, S. Sachdev, M. Greiner, V. Vuletic, and M. D. Lukin, arXiv:2012.12281 .

[11] D. Bluvstein, A. Omran, H. Levine, A. Keesling, G. Semeghini, S. Ebadi, T. T. Wang, A. A. Michailidis, N. Maskara, W. W. Ho, S. Choi, M. Serbyn, M. Greiner, V. Vuletic, and M. D. Lukin, Science 371, 1355 (2021).

[12] S. de Léséleuc, V. Lienhard, P. Scholl, D. Barredo, S. Weber, N. Lang, H. P. Büchler, T. Lahaye, and A. Browaeys, Science 365, 775 (2019).

[13] C. J. Turner, A. A. Michailidis, D. A. Abanin, M. Serbyn, and Z. Papić, Nat. Phys. 14, 745 (2018).

[14] C. J. Turner, A. A. Michailidis, D. A. Abanin, M. Serbyn, and Z. Papić, Phys. Rev. B 98, 155134 (2018).

[15] A. J. A. James, R. M. Konik, and N. J. Robinson, Phys. Rev. Lett. 122, 130603 (2019).

[16] N. Shibata, N. Yoshioka, and H. Katsura, Phys. Rev. Lett. 124, 180604 (2020).

[17] S. Sachdev, Quantum Phase Transitions. Second edition (Cambridge University press, Cambridge, U.K., 2011).

[18] S. Suzuki, J.-i. Inoue, and B. K. Chakrabarti, Quantum Ising Phases and Transitions in Transverse Ising Models (Springer,
Berlin/Heidelberg, 2013).

[19] P. Pfeuty, Ann. Phys. 57, 79 (1970).

[20] F. Robicheaux and J. V. Hernández, Phys. Rev. A 72, 063403 (2005).

[21] H. W. J. Blöte and Y. Deng, Phys. Rev. E 66, 066110 (2002).

[22] R. Moessner, S. L. Sondhi, and P. Chandra, Phys. Rev. Lett. 84, 4457 (2000).

[23] R. Moessner and S. L. Sondhi, Phys. Rev. B 63, 224401 (2001).

[24] P. Sen, Phys. Rev. E 63, 016112 (2000).

[25] O. F. de Alcantara Bonfim, B. Boechat, and J. Florencio, Phys. Rev. E 99, 012122 (2019).

[26] M. A. Novotny and D. P. Landau, J. Magn. Magn. Mater. 54-57, 685 (1986).

[27] A. A. Ovchinnikov, D. V. Dmitriev, V. Y. Krivnov, and V. O. Cheranovskii, Phys. Rev. B 68, 214406 (2003).

[28] Y. Motoyama, K. Yoshimi, A. Masaki-Kato, T. Kato, and N. Kawashima, Comput. Phys. Commun. 264, 107944 (2021).

[29] J. Gubernatis, N. Kawashima, and P. Werner, Quantum Monte Carlo Methods: Algorithms for Lattice Models (Cambridge University press, Cambridge, U.K., 2016).

[30] S. El-Showk, M. F. Paulos, D. Poland, S. Rychkov, D. SimmonsDuffin, and A. Vichi, J. Stat. Phys. 157, 869 (2014).

[31] Y. Kato and T. Misawa, Phys. Rev. B 92, 174419 (2015).

[32] K. Harada, Phys. Rev. E 84, 056704 (2011).

[33] H. Rieger and N. Kawashima, Eur. Phys. J. B 9, 233 (1999).

[34] C. Domb, Adv. Phys. 9, 245 (1960).

[35] J. Wurtz and A. Polkovnikov, Phys. Rev. B 101, 195138 (2020).

[36] P. Lajkó and F. Iglói, Phys. Rev. B 103, 174404 (2021).

[37] F. Iglói, Phys. Rev. B 40, 2362 (1989).

[38] Y.-P. Lin, Y.-J. Kao, P. Chen, and Y.-C. Lin, Phys. Rev. B 96, 064427 (2017).

[39] P. Lajkó, J.-C. A. d'Auriac, H. Rieger, and F. Iglói, Phys. Rev. B 101, 024203 (2020).

[40] R. Samajdar, W. W. Ho, H. Pichler, M. D. Lukin, and S. Sachdev, Phys. Rev. Lett. 124, 103601 (2020).

[41] N. Kellermann, M. Schmidt, and F. M. Zimmer, Phys. Rev. E 99, 012134 (2019).

[42] X. Wang, M. H. Christensen, E. Berg, and R. M. Fernandes, arXiv:2102.11985.

[43] R. Samajdar, W. W. Ho, H. Pichler, M. D. Lukin, and S. Sachdev, Proc. Natl. Acad. Sci. U.S.A. 118, e2015785118 (2021). 